\title{
Venus and Freud: an educational opportunity?
}

\author{
G Lloyd Jones, J G Bligh, C Valentine
}

Objective: To discuss the importance of genitourinary medicine as a core component for a new problem based medical undergraduate curriculum and to describe the advantages of consensus group method as a means of identifying learning objectives for an integrated course.

Methods: A group of experts in the field of sexual health was convened around the module on sexual health. It was led by a genitourinary specialist and aided by a curriculum facilitator. Group contributors represented a wide variety of disciplines and included a medical student. The group met on three occasions for 1.5 hours.

Results: The identified learning objectives are presented under the course themes. The values and relevance of the individual objectives are discussed with particular reference to an integrated curriculum which combines clinical and basic science in a problem based learning (PBL) format. The communication skills and attitudes necessary to take a competent sexual history are stressed. Links with other relevant modules in the PBL curriculum are described to show how the topic of sexual health develops throughout the course. Learning methods, resources and assessment procedures are also outlined.

Conclusion: The educational potential of sexual health and behaviour as a core topic for a novel integrated course is considerable and merits a greater importance than it appears to receive in current undergraduate courses.

(Genitourin Med 1996;72:290-294)

Keywords: sexual health; education; curriculum design

The University

Medical Education

Unit, The University of

Liverpool, 3rd Floor,

UCD Building, Royal

Liverpool University

Hospital, Liverpool,

L69 3BX, UK

G Lloyd Jones

J G Bligh

Department of Medical Microbiology

and Genitourinary

Medicine, The

University of Liverpool

C Valentine

Correspondence to:

G Lloyd Jones.

Accepted for publication

16 May 1996

\section{Introduction}

Concern has been voiced about the declining importance of genitourinary medicine in undergraduate medical curricula in the UK. Cowan and Adler's study ${ }^{1}$ reported an overall reduction in the amount of time devoted to genitourinary medicine in the period between 1984 and 1989, despite the prominence of HIV and AIDS during this time. Their study revealed widespread regional variation, one school offering no genitourinary medicine and another where it became optional shortly after the questionnaire was returned. This concern has prompted the Education sub-committee of the Medical Society for Venereal Diseases to review aspects of undergraduate teaching of GUM in the United Kingdom. ${ }^{2}$

Teaching time, one of the measures used in Cowan and Adler's report, ${ }^{1}$ is not the sole arbiter of quality in an educational programme nor is it the most apposite. In the wake of the report, Peters and Weber ${ }^{3}$ challenged teachers to devise novel and imaginative courses based on learning objectives, self-directed learning and appropriate assessment procedures.

Currently a number of external factors are influencing the delivery of Sexual Health services in the United Kingdom towards greater integration to reduce multiple attendances and maximise effective use of resources. ${ }^{4}$ These changes in the structure of NHS genitourinary medicine services should be reflected in how sexual health is learned by undergraduates.

Likewise the General Medical Council's document Tomorrow's Doctors ${ }^{5}$ provides a further stimulus to educational reform at undergraduate level. Amongst the GMC's main recommendations were a reduction in factual content and the promotion of an integrated curriculum. These ideals run counter to the trend towards greater specialisation within both academic and clinical medicine with its dependence on an ever expanding knowledge base.

Superficially, there is a paradox between these, apparently irreconcilable, aims. Continually pruning core content will only be counterproductive in the long term. What is needed is a more radical approach which ultimately questions the basic assumptions underlying current undergraduate courses.

In 1996 the medical school at Liverpool University will introduce an integrated problem-based course along the lines advocated by the GMC. This paper describes the processes involved in meeting this educational challenge in the field of sexual health.

\section{Overview of the Course}

The new Liverpool PBL course is modular, each module focusing on a clinical topic. There are 58 modules in all, running throughout the first 4 years of the course. Each module lasts one or two weeks and, during the first 3 years, all students progress through them simultaneously.

The first year modules have a basic science bias but are presented via commonplace clinical scenarios. In years $2-4$ the module topics mirror the life-cycle beginning with conception, genetics, normal pregnancy and birth and moving on through child development to puberty. The module on sexual health and behaviour takes place in the second year of the course and follows these modules (table 1).

Normality is the focus of the first life-cycle 
Table 1 Module Sequence-1st Life-Cycle

\begin{tabular}{cl}
\hline 1. & Contraception \\
2. & Pregnancy \\
3. & Birth and Lactation \\
4. & The Neonate \\
5. & Clinical Genetics \\
6 and 7. & Child Development \\
8. & Common Childhood Infections \\
9. & Puberty \\
10. & Drug Abuse \\
11. & Sexual Health and Behaviour \\
\hline
\end{tabular}

(years 2 and part of 3 ) and encourages the student to develop a thorough understanding of biological and behavioural normality as a sound basis for diagnostic ability. Accompanying this is an emphasis on the acquisition of communication skills necessary for history taking.

The sexual health theme is developed later in the PBL course by incorporating it within specific clinical scenarios in related modules, for instance, pelvic inflammatory disease in the Acute Abdominal Pain and Infertility modules. Later modules dealing with psychiatric disorders and palliative care provide further examples of ways in which themes can be unfolded within an integrated PBL curriculum. Figures 1 and 2 demonstrate the modular distribution of sexual health within years $2-4$ of the course.

Four themes permeate the PBL curriculum. These are: Structure and Function in Health and Disease, Individuals, Groups and Society, Public Health and Epidemiology and Professional Values and Ethics. The Structure and Function theme incorporates basic science, pharmacology and pathology. Individuals, Groups and Society covers the relevant areas of psychology and sociology. The Public Health theme includes epidemiology, economics and health promotion. The Professional Values and Ethics theme introduces topical and controversial issues surrounding current medical practice.

\section{Delivery of the Curriculum}

The PBL curriculum will be delivered via a mixture of plenary sessions, small group work and computer assisted learning with a strong leaning towards independent, self-directed learning methods. Students will work in groups of six facilitated by a tutor and sup-

Figure 1 Modular distribution of sexual health in year 2 .

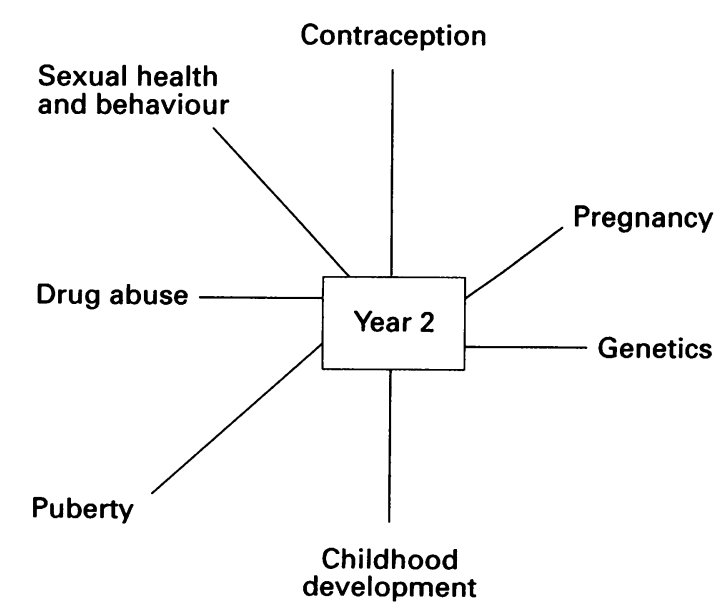

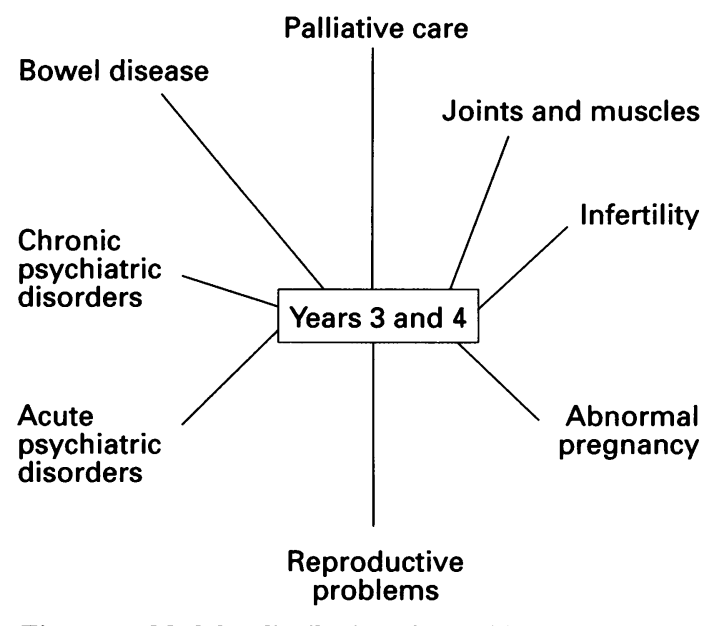

Figure 2 Modular distribution of sexual health in years 3 and 4.

ported by guides which include self-assessment questions and references.

As the new course is modelled on a core and options approach all students will have the opportunity to study specialist disciplines in greater depth in six special study modules between second and final year. Each special study module lasts four weeks and all specialities within faculty have been invited to produce suitable programmes in conjunction with the Medical Education Unit.

Assessment takes place in different forms throughout the course. The student guides and computer aided learning (CAL) software accompanying the modules include selfassessment questions which provides on-going assessment. Summative assessments will take place at the end of each semester.

The core Sexual Health and Behaviour module has been allocated one week in the first life-cycle. It is protected from overloading by the life-cycle structure of the course. Students concentrate on the life science foundations of reproduction and development in the modules on conception, pregnancy and puberty which precede the Sexual Health and Behaviour module. As a result they are encouraged to revisit and extend basic science knowledge into the behavioural field and enter this module with extensive prior knowledge.

It is important that links are clearly established between topics in an integrated PBL curriculum to avoid a "patchwork" effect with lack of continuity, sequence and structure. In practice the study guides and CAL software will draw the students' attention to links between related subject areas. Figure 1 displays these linkages for the second year of the PBL course and figure 2 for years 3 and 4 . The core module is just one element of an extended sexual health curriculum which students encounter over the four years of the PBL course.

The clinical course runs simultaneously with the PBL curriculum and is shared between hospital and community sectors. Whilst students and clinical teachers will be encouraged to concentrate on presenting cases 
relevant to the PBL topic for that week it is unlikely that the two courses can be combined more closely than this. To allow for this eventuality, provision is made in the time-table for students to attend GUM clinics at fixed times during the course. The PBL course ceases at the end of fourth year leaving the final year as a clinical apprenticeship.

As part of the clinical course each student will be given a clinical log-book detailing 93 core cases which all students must see prior to graduation. Included in this list are cases of AIDS and urethral discharge. Students will be expected to have mastered the pathophysiology, diagnosis and management of these cases.

\section{Curriculum planning}

The first major decision was to allow the topic of sexual health and behaviour a module of its own so establishing it as an essential part of the core curriculum. The design of the course around the life-cycle pattern, rather than the more conventional systems-based structure, fosters the inclusion of sexual health as a separate topic. In systems-based courses sexual health may become a mere adjunct of the physical sciences whereas in the life-cycle structure it falls naturally within a group of behaviourally orientated topics such as puberty, drug abuse and other related lifestyle issues such as exercise and nutrition.

However there were several other reasons for its inclusion. Firstly, its importance within medical practice is extensive, reaching well beyond the boundaries of genitourinary medicine to obstetrics and gynaecology, primary care, family planning and psychiatry. In the genitourinary field alone, numbers of new cases reporting to STD clinics rose from 560159 in 1988 to 656902 in $1992 .^{6}$ No comparable data on the caseload seen in general practice or family planning clinics are available but they are likely to be similar.

The Health of the Nation ${ }^{7}$ identified a number of priorities in related aspects of sexual health including reductions in the incidence of teenage pregnancy and gonorrhoea rates. It also advocated controlling the spread of HIV and AIDS through health education. Responsibility for this was placed, at the local level, on primary care teams and genitourinary medicine specialists.

The importance of sexuality in people's lives is beyond doubt, a fact long and successfully acknowledged by the advertising industry. There is a dearth of evidence surrounding the medical student's own knowledge and attitudes in this area and this also played a part in the choice of sexuality as part of core.

The essential privacy of sexual experience brings with it problems of its own, not least the confidentiality which permeates medical practice in this field. Whilst public opinion sanctions considerably more openness on sexual matters than previously the experiential nature of sexuality remains a social taboo. Consequently, attitudes play a significant role in any educational process concerned with sexual health. To deal adequately with this problem students need to acquire sensitive communica-
Table 2 Consensus group contributors by speciality

\begin{tabular}{ll}
\hline Convenor & Genitourinary Medicine \\
Contributors & Family Planning \\
& Genitourinary medicine \\
& Nursing \\
Obstetrics and Gynaecology & Primary Care \\
& Physiology \\
& Psychiatry \\
& Public Health \\
& Medical Student \\
Facilitator & Medical Education Unit \\
\hline
\end{tabular}

tion skills and an awareness of the ethics of professional responsibility. They should also have an understanding of how attitudes towards sexuality change with time and circumstance within society and of the complex relationship between attitudes and behaviour in this area.

Finally, sexual behaviour provides an excellent example of how behaviour is determined by both biological and social forces and the difficulties involved in any analysis of these separate influences.

\section{Method}

Consensus group method was used to identify the learning objectives, core and clinical skills surrounding sexual health and behaviour. ${ }^{8}$ Learning objectives specify what knowledge the student needs to acquire in an assessable format; for instance, students should be able to outline how sexually transmitted diseases impact on other areas of health. The objectives and core then form the basis of a new curriculum.

A group of experts was convened and led by a lecturer of genitourinary medicine. The group was facilitated by a medically qualified research fellow based in the Medical Education Unit. Contributors to the group came from various disciplines and included representatives from family planning, obstetrics and gynaecology, nursing, physiology, primary care, psychiatry, public health and the student body (table 2). Their joint task was to identify the specific learning objectives over a period of three meetings of around 1.5 hours each. The group also had to consider the appropriate level of complexity for second year students bearing in mind the topics already covered. They also suggested a framework for the structured teaching within the Sexual Health and Behaviour module.

\section{Results}

After lively discussion the group agreed the learning objectives listed in table 3 and the following outline for the module. At the start of the module week the students are presented with a clinical scenario which focuses the student's attention on the learning objectives. The scenario starts with a new patient attending an GUM clinic with the quote "My boyfriend has given me this contact slip and suggested I come to the clinic". After the initial group meeting at which the students identify their own learning objectives there are four 
Table 3 Learning objectives-Sexual Health and Behaviour module

Structure and Function in Health and Disease and Individuals, Groups and Society

Describe the anatomy of the reproductive systems in both sexes.

- Describe the sexual responses both physiologically and emotionally.

Examine the nature of sexuality.

Analyse the factors influencing sexual behaviour.

Question the concept of normality in relation to sexual behaviour.

Discuss the influence of sex education on sexual activity.

- List the current 5 commonest sexually transmitted diseases: a. in the UK

Outline how sexually transmitted diseases impact on other areas of health.

What are the economic implications of STD's?

Public Health and Epidemiology

Analyse the problems associated with cross-sectional studies with reference to health

- Examine the cohort, period and age effect in relation to STD's eg cervical cancer,

chlamydia, hepatitis B, syphilis and HIV.

- Explain validity in relation to screening for cervical cancer.

Professional Values and Ethics

Identify the legal framework surrounding sexual behaviour.

Debate the nature of confidentiality in the field of sexual health care.

- Explain how the specific regulations of the VD Act, Public Health legislation and Children Act and laws concerning sexual behaviour might cause dilemmas for the practitioner with the right of the individual to confidentiality.

Clinical Skill

- To be able to take a sexual history.

periods during the week for self-study. At the end of this week the group meet with their tutor to satisfy themselves that the objectives have been achieved and the case adequately explained.

There are three plenary sessions associated with this module, the first an introductory lecture on the history of sexually transmitted diseases to set the scene and highlight the importance of cultural norms and legislation on sexual behaviour. The second session entitled "Where do our attitudes come from?" covers psychosocial issues which provides the ground work for the development of communication skills. The final session debates the nature of confidentiality in the field of sexual health care. CAL will guide the students to revise and update knowledge already acquired in earlier modules.

Taking a sexual history was identified by the consensus group as an essential skill. As the group felt few students were comfortable with role play in this situation they recommended the employment of a standardised patient. This has the added advantage of allowing interviews to be videotaped with improved assessment and feedback but may present resource problems.

These new arrangements will constitute a considerable increase in the statutory time allocated for core genitourinary medicine teaching from the current 27 hours on the present course.

\section{Discussion}

These results demonstrate how genitourinary medicine can provide an effective and imaginative core element for an integrated curriculum and, in particular, how sexual health and behaviour constitutes an excellent and provocative educational vehicle. The consensus group method has proved an effective means of identifying the educational core in the development of a new course particularly when integration is a basic principle.

Although under the new system the time allocated for teaching of GUM has increased it is difficult to define this with precision. The integrated curriculum renders interdisciplinary boundaries less distinct, especially so within the context of self-directed learning. Much of the teaching conventionally undertaken by pre-clinical departments such as psychology and sociology has been incorporated into our scheme. It is likely that the four medical schools considered by the MSSVD report ${ }^{2}$ will likewise not have recognised this aspect of teaching of GUM related matters in their institutions.

Inclusion of sexual health and behaviour as a core component of the curriculum reinforces its importance in wider areas of health and disease both for students and other members of medical faculty who may perceive it as a low priority area undeserving of increased teaching time.

Like one of the other provincial medical schools ${ }^{2}$ teaching of genitourinary medicine in Liverpool has previously been "tagged on" to the obstetrics and gynaecology attachment. This has frequently caused problems for students who were unable to attend the genitourinary medicine clinic because of other commitments that they perceived as more pressing. By allocating protected time for sexual health and behaviour we hope to remove some of this pressure and ensure that all Liverpool graduates are able to spend one full week studying a range of sexual health issues.

In common with Cowan and colleagues, the group identified competence in taking a sexual history as a key clinical skill but did not consider it desirable or appropriate for a newly qualified doctor to undertake a genital examination in screening for sexually transmitted diseases. This was agreed to be a requirement more suitable for the postgraduate level in the absence of the facilities, resources and expertise provided by the genitourinary clinic.

The group decided to combine the biological and psychosocial themes for this module because of the impossibility of isolating their separate influences on sexual behaviour. The linking of the two themes demonstrates the strength of integration in providing contextually appropriate and demanding learning material.

Analysing the objectives in more detail reveals what can be extracted educationally using this approach. The anatomy and physiology objectives are revisional as they have already been introduced earlier in the course. Revisiting concepts is essential to reinforce learning and extend knowledge but may present problems to curriculum planners who have to pay attention to sequencing and unfolding complexity. The new material in this module consists of the commoner sexually transmitted diseases and their consequences and implications for health.

There is also a broad emphasis on the behavioural objectives which links with communication skills. During the first year of the new course the students receive practical instruction in basic communication skills which will prepare them for the clinical skil 
outlined by the consensus group in this module namely, taking a sexual history. Good history taking demands not only sensitivity and discretion, but an awareness of the implicit assumptions underlying one's own sexuality and how this may affect the management of a patient of differing sexual orientation. The behavioural objectives may be seen as challenging but sexual health is an area where attitudes are crucial to good medical practice. The broader message for both educators and students alike is to take nothing for granted.

The objectives under the Public Health heading cover a wide range of issues including the nature and value of cross-sectional studies, the principles underlying screening procedures and the statistical analysis of epidemiological data.

Finally, the objectives under the last theme introduce sociological and legal issues reminding students of the doctor's role within society at large. Confidentiality and the conflicts that may arise from the doctor's dual responsibilities to patient and state form the basis of this theme.

\section{Conclusion}

The model described represents a successful, collaborative attempt to meet the challenge set by Tomorrow's Doctors in the field of sexual health. It is not suggested that the model is prescriptive and we anticipate some evolution as development gathers pace. Implementation will doubtless bring further amendments. It is more than likely that other medical schools will select different themes, emphases and sequencing with alternative and satisfactory results. However, it does serve as an example of what may be achieved by corporate effort when there is determination, imagination and willingness to change. A vital key to success is the readiness to challenge long cherished assumptions, however uncomfortable this may be. Only once this principle is accepted can the virtues of a novel educational alliance between Venus and Freud be fully realised.

The members of the consensus group were Dr C Valentine, $\mathrm{Dr}$ A Alawattegama, Dr R Banks, Mr G Carr, Dr C Dowrick, Dr B Friedman, Dr MJ Platt, Mr P Rose, Dr D Taylor, Dr N Chater and Miss A Garden.

1 Cowan FM, Adler MW. Survey of undergraduate teaching in genitourinary medicine in Britain. Genitourin Med 1994;70:311-3.

2 Cowan FM, Dhar J, Patel R, Ainsworth J, Peters B, Bingham J. Genitourinary medicine in Britain-what are the issues? Genitourin Med 1996;72:6-8.

3 Peters BS, Weber JN. Undergraduate teaching in genitourinary medicine. Genitourin Med 1994;70:299.

4 Kubba A. Consensus workshop on sexually transmitted diseases and contraception: sexual health promotion and service delivery. Br $\mathcal{F}$ Family Planning (in press).

5 General Medical Council. Tomorrow's Doctors. Report of the Education Committee 1993

6 Department of Health. Health and Personal Social Services Statistics for England. London HMSO 1994:122.

7 Secretary of State for Health. The Health of the Nation. London HMSO 1991.

8 Bligh J. Identifying the core curriculum: the Liverpool approach. Medical Teacher 1995;17:383-90. 